\title{
Evolución de la hidratación en cementos con adiciones
}

\section{Evolution of the hydration in cements with additions}

V. L. BONAVETTI, V. F. RAHHAI, E. F. IRASSAR

Departamento de Ingeniería Civil - Facultad de Ingeniería - Universidad Nacional del Centro

Olavarría-Argentina

Fecha de recepción: 5-VI- 02

Fecha de aceptación: 30-VIII-02

ARGENTINA

\begin{abstract}
RESUMEN
En el presente trabajo se analizó el mecanismo de hidratación de pastas de cemento portland normal con la incorporación de caliza, cuarzo y puzolana natural (proporción 80/20 en peso), por medio de la evaluación del contenido de agua no evaporable, la actividad puzolánica por vía química y la formación de productos de hidratación por DRX. Los resultados obtenidos permitieron determinar un aumento de la cantidad de productos de hidratación inicial debidos al efecto filler en todas las pastas. En las pastas con caliza se evidenció el efecto de dilución a edades aranzadas, en tanto que en las pastas con cuarzo y. puzolana, este efecto fue menos predominante debido a la contribución de la reacción puzolánica.
\end{abstract}

\begin{abstract}
SUMMARY
In this paper; the hydration mechanism of portland cement pastes with limestone, quartz and natural pozzolan (80/20) weight) was analized. The techniques used were nonevaporable water content, pozzolanic activity and X-ray. diffraction. Results show that filler effect increases initially. the amount of hydration products in all pastes. For limestone pastes, the dilution effect is significant at long time, for quartz and pozzolan pastes the dilution effect was lower due to the contribution of the pozzolanic reaction.
\end{abstract}

PALABRAS CIAAVE: caliza, cuarzo, puzolana, agua no cvaporable, actividad puzolánica, DRX

\section{INTRODUCCIÓN}

Cuando se incorpora una adición mineral a un cemento portland, se producen tres efectos, que dependiendo de las proporciones en que se encuentren mezclados los matcriales, y de sus características físicas y químicas, varían su importancia en función del tiempo. Ellos son: el efecto de dilución del cemento portland, presente durante toda su hidratación; el efecto filler, preponderante en las primeras edades de la misma; y cuando la adición la presenta. la actividad puzolánica, que, según su grado de reactividad, puede ser fuerte, moderada o débil. Las con-
KEYWORDS: limestone, quartz, pozzolan, non evaporable water, pozolanic activity; $X R D$

secuencias negativas del efecto de dilución, provocado por incorporación de una adición mineral al cemento portland sobre las propiedades mecánicas de la pasta, el mortero o el hormigón, tiende a ser compensado en principio por el efecto filler y, posteriormente, por la actividad puzolánica cuando ésta es capaz de desarrollarla. El grado de compensación entre estos efectos. depende fundamentalmente de la reactividad (morfología, mineralogía, tamaño) y de la cantidad de adición.

La incorporación de caliza molida como reemplazo parcial del clínker genera dos efectos sobre la hidratación 
del mismo: por un lado el efecto químico que tiene como consecuencia la formación de carboaluminatos de calcio hidratado (1-2) y por otro, el efecto físico que provoca la dispersión de los granos de cemento acelerando la velocidad de hidratación del mismo (3). El efecto de dispersión se atribuye a la ubicación de la adición entre los granos de cemento, que aumenta su área de contacto con el agua, y posibilita un aumento en la velocidad de hidratación inicial. Además, este incremento también se atribuye a que las partículas de caliza actúan como centros de nucleación alrededor de las cuales se depositan los cristales de hidróxido de calcio (CH) (3-4). Desde el punto de vista de la resistencia, el efecto físico es el principal responsable del aumento de este parámetro a edades tempranas (5).

La incorporación de cuarzo finamente molido, produce un efecto filler similar al que produce la caliza molida (6), por este motivo se considera que estas adiciones sólo colaboran con la resistencia inicial del cemento y que las mismas deben ser consideradas como parte integrante de la arena. En contrapartida, otros autores $(4,7-9)$ consideran que a pesar de la elevada cristalinidad que posee el cuarzo, puede contener en la superficie alguna cantidad de matcrial vítreo, y/o que parte de este material cristalino puede disolverse en un medio altamente básico (como lo es la pasta de cemento), reaccionando en ambos casos con el $\mathrm{CH}$ para producir una reacción puzolánica a edades avanzadas de la hidratación. En este sentido, Massazza y Pezzuoli (8) registraron un importante aumento de la dureza de la zona de interface pasta de cemento-cuarzo, atribuyendo este incremento a las reacciones que se producen entre ambos materiales.

El empleo de puzolana natural como adición en el cemento portland ocasiona principalmente la formación de silicato de calcio hidratado $(\mathrm{C}-\mathrm{S}-\mathrm{H})$ de características similares al que se produce durante la hidratación de los silicatos de calcio del clínker portland. La reacción es relativamente lenta, razón por la cual la ganancia de resistencia asociada es diferida en el tiempo. Con el progreso de la reacción, además de la manifestación química habrá una manifestación física que consistirá en un refinamiento de poros en la pasta de cemento. Por otra parte, la incorporación de puzolana, también produce efecto filler (10) en las primeras edades de la hidratación, pero dependiendo del contenido de la misma en el cemento puede ser superado por el efecto de dilución.

En el presente trabajo se analizan las modificaciones en el mecanismo de hidratación de pastas de cemento portland normal con caliza, cuarzo y puzolana natural (proporción 80/20 en peso) debidas al efecto de dilución, al efecto filler y a la actividad puzolánica. Las técnicas utilizadas en la evaluación fueron: contenido de agua no evaporable, actividad puzolánica por vía química y formación de productos de hidratación por DRX.

\section{MATERIALES Y METODOLOGÍAS DE ENSAYO}

Los matcriales empleados para la realización de los estudios fueron: un cemento portland normal (CPN) compuesto por $51,2 \%$ de $\mathrm{C}_{3} \mathrm{~S}, 25,9 \%$ de $\mathrm{C}_{2} \mathrm{~S}, 5,6 \%$ de $\mathrm{C}_{3} \mathrm{~A}$ y 9,8\% de $\mathrm{C}_{4} \mathrm{AF}$, de clase resistente CP40 (IRAM 50000, $40 \mathrm{MPa}$ a 28 días), con un parámetro de homogeneidad de 0,9 y de posición de $26,6 \mu \mathrm{m}$, obtenidos a partir de la curva de distribución de las partículas y una finura Blaine de $285 \mathrm{~m}^{2} / \mathrm{kg}$. Tres adiciones minerales, una caliza molida (F) con $85 \%$ de $\mathrm{CaCO}_{3}$ y como principal impureza $\mathrm{SiO}_{2}$; un cuarzo molido (Q) con $99,99 \%$ de $\mathrm{SiO}_{2}$ y una puzolana natural molida $(\mathrm{P})$ con una pequeña fracción de cuarzo y feldespatos (anortita, albita, chabazita y analcita). Las adiciones estudiadas fueron molidas para obtener una superficic específica Blaine comprendida entre 710 y $760 \mathrm{~m}^{2} / \mathrm{kg}$.

Proporciones de las muestras: con cstos materiales se prepararon mezclas en proporción en peso 80/20 cemento/adición, a partir de las cuales se elaboraron pastas con relación agua/material cementante $(\mathrm{a} / \mathrm{mc})$ de 0,40 . La relación agua/cemento necesaria para lograr la hidratación total de un cemento portland varía de acuerdo a su composición y a su finura entre 0,21 y 0,33 (11); sin cmbargo, esta cantidad de agua no provee el cspacio necesario para que se desarrollen los productos de hidratación. Se ha comprobado que la relación agua/cemento mínima para que una pasta de cemento no sellada pueda alcanzar la hidratación total es del orden de 0,36 a 0,38 (11). Con el objeto de permitir que la muestra con cemento CPN pueda alcanzar esta condición y que la falta de espacio para alojar los productos de hidratación no fuera un parámetro que modificara el desarrollo de la misma, se adoptó una relación a/mc de 0,40 . Con las pastas antes mencionadas se moldearon probetas prismáticas que permanecieron 24 horas en el molde, y luego de desmoldadas, se sumergicron en agua saturada con cal hasta las edades de ensayo $(1,3,7,28,90$ y 180 días $)$.

Agua no evaporable: la cantidad de agua no evaporable sc determinó de acuerdo al procedimiento propuesto por Powers (12). Este valor se utilizó como estimador del progreso de la reacción de hidratación, asumiendo que la caliza molida y el cuarzo molido son hidráulicamente inactivos y que la totalidad de la puzolana natural molida incorporada es capaz de reaccionar para producir C-S-H.

Puzolanicidad: para complementar este estudio se evaluó la actividad puzolánica por vía química a los 28 y 90 días. El análisis se realizó mediante la comparación de la cantidad de hidróxido de calcio que contenía la disolución acuosa de cada pasta hidratada, con la isoterma de solubilidad de hidróxido de calcio en una disolución alcalina. La temperatura de ensayo fue de $40^{\circ} \mathrm{C}$. El resultado del ensayo determina actividad puzolánica, cuando 
la concentración de hidróxido de calcio en la disolución acuosa de cada pasta, se ubica por debajo de la isoterma de solubilidad.

Resistencia mecánica: la resistencia a flexión de las pastas se evaluó sobre probetas prismáticas, con una sección transversal de $25 \times 25 \mathrm{~mm}$ y una luz entre apoyos de $62,5 \mathrm{~mm}$ con carga centrada. Sobre los fragmentos resultantes de esta determinación se realizó el ensayo a compresión. Los resultados informados corresponden al promedio de cuatro y ocho determinaciones por edad, respectivamente.

Difracción de Rayos X (DRX): Las determinaciones se realizaron con un difractómetro Philips X'Pert equipado con monocromador de grafito, usando radiación $\mathrm{CuK} \alpha, \mathrm{y}$ operando a $40 \mathrm{kV}$ y $20 \mathrm{~mA}$. El registro se realizó a una velocidad de $2 \% \mathrm{~min}$ y el intervalo de paso fue de $0,02^{\circ}$. $\mathrm{El}$ análisis semicuantitativo del hidróxido de calcio $(\mathrm{CH})$ se realizó integrando el área bajo el pico de $2 \theta^{\circ}=18,09^{\circ}$, $\mathrm{d}=4,90 \mathrm{~nm}$.

\section{RESULTADOS Y DISCUSIÓN}

Agua no evaporable: la evolución del agua no evaporable relativa de las pastas con adiciones a la pasta CPN, se muestra en la Figura 1. Los resultados fueron calculados descontando a la caliza y al cuarzo del material cementante y considerando a la totalidad de la puzolana como material cementante. En la Figura 1 puede observarse que las pastas con caliza y cuarzo molido, hasta los 28 días presentaron un comportamiento similar; registrando la máxima ganancia de agua no evaporable con respecto al patrón (17 al $19 \%$ ) entre los 3 y los 7 días. Este comportamiento puede justificarse a partir del efecto filler, pues la incorporación de materiales finamente molidos completa la distribución granulométrica del clínker (13), creando una mayor cantidad de núcleos a partir de los cuales comienza el crecimiento del hidróxido de calcio (3-4). En función de lo expuesto y desde el punto de vista del agua no evaporable, hasta los 28 días la incorporación de caliza y cuarzo molidos producen el mismo efecto de aceleración de la hidratación sobre el clínker portland (6).

A partir de los 28 días el comportamiento de cada una de estas pastas comenzó a diferenciarse. En la pasta $\mathrm{CPN}+20 \mathrm{~F}$, la cantidad relativa de agua no evaporable empezó a disminuir alcanzando, a la edad de 180 días, a ser sólo del $5 \%$. Con el transcurso del tiempo y la reacción del clínker de la pasta CPN, los niveles de agua no evaporable comienzan a igualarse, perdiéndose el efecto inicial de aceleración de las reacciones (14) producidas en la pasta $\mathrm{CPN}+20 \mathrm{~F}$.

Por su parte, en la pasta CPN+20Q a partir de los 28 días, el contenido de agua no evaporable relativo a la pasta CPN, comenzó a aumentar, alcanzando a ser $17 \%$ mayor a la edad de 180 días, indicando que ha ocurrido algún tipo de reacción que produjo una mayor cantidad de productos de hidratación. El cuarzo molido, que posee una estructura bien cristalizada, puede probablemente presentar una estructura amorfa en la superficie generando una reacción puzolánica (7-9) entre la superficie de la adición y el $\mathrm{CH}$ liberado por la hidratación de los silicatos de calcio del clínker portland.

En el caso de la pasta $\mathrm{CPN}+20 \mathrm{P}$, en la Figura 1 puede observarse que las adiciones activas también producen efecto filler (10), el cual se encuentra representado por el incremento del agua evaporable relativa hasta la edad de 3 días. A partir de esta edad, el efecto de dilución se torna más importante hasta que comienza la reacción de la puzolana, permitiendo, a la edad de 180 días, que este parámetro sea del mismo orden del control.

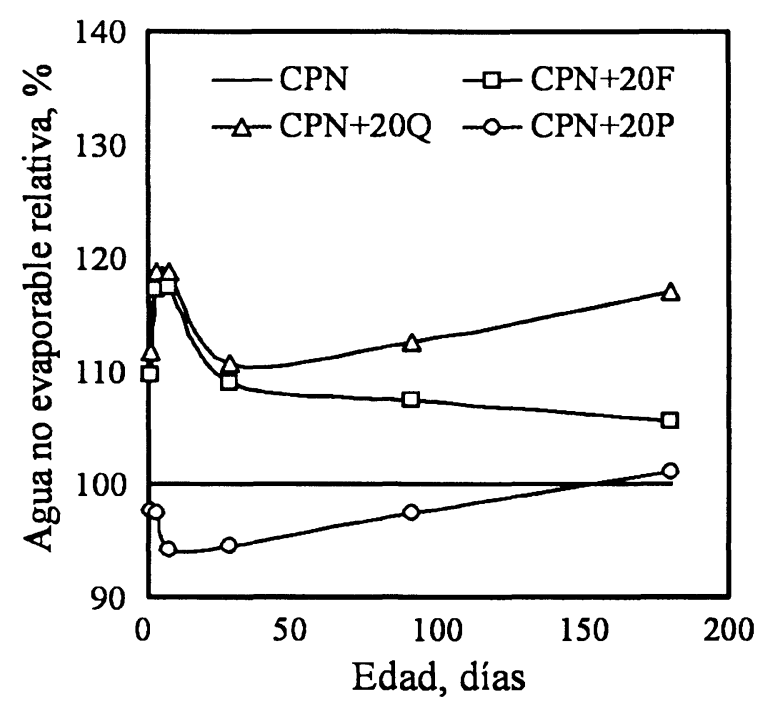

Figura 1.- Evolución de la cantidad del agua no evaporable relativa a la pasta CPN.

Puzolanicidad: en la Figura 2 se observan las posiciones de los puntos representativos de las muestras estudiadas con respecto a la isoterma de solubilidad a los 28 y 90 días. De acuerdo a los resultados obtenidos se puede confirmar que la incorporación de caliza molida provoca una aceleración de la hidratación de los silicatos debido a la mayor generación de $\mathrm{CH}$ (zona de sobresaturación). Además, puede apreciarse que el cuarzo molido posee una tendencia a la actividad puzolánica, dada por la evolución del punto representativo de la zona de sobresaturación a los 28 días, a la zona de subsaturación a los 90 días. Por último la pasta con $20 \%$ de puzolana ha mostrado actividad puzolánica en ambas edades.

Resistencia mecánica: en la Figura 3 se muestra la evolución de la resistencia a flexión de las pastas estudiadas. La pasta $\mathrm{CPN}+20 \mathrm{~F}$ presentó una reducción de la resistencia de $20 \%$ con respecto a la de la pasta CPN a la edad de un día. Entre los 7 y 180 días, esta disminución permanece en el orden de $4 \%$. Por su parte, la pasta 


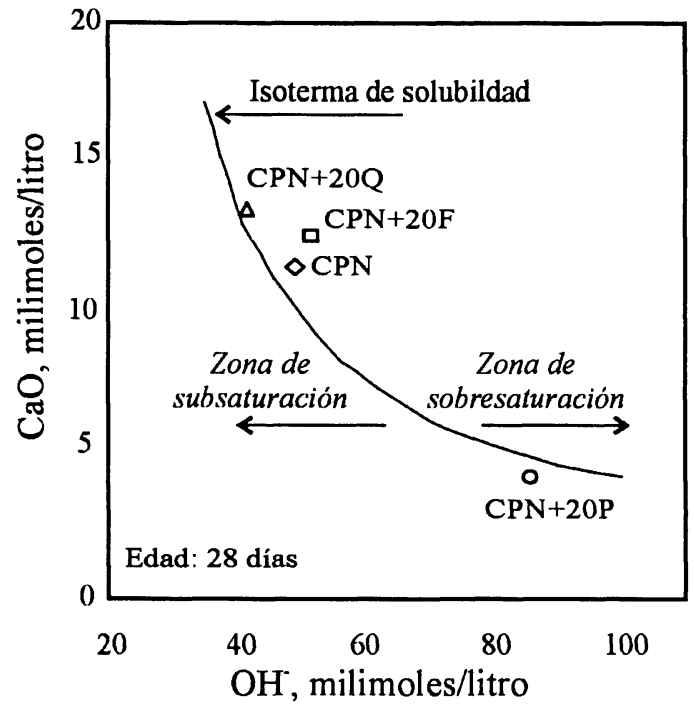

a)

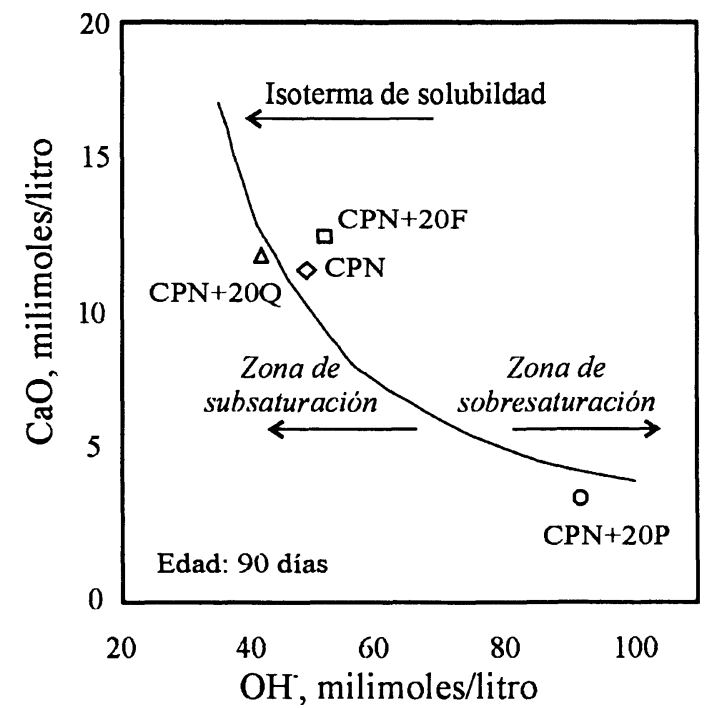

b)

Figura 2.- Actividad puzolánica por vía química de los materiales utilizados: a) 28 días y b) 90 días.

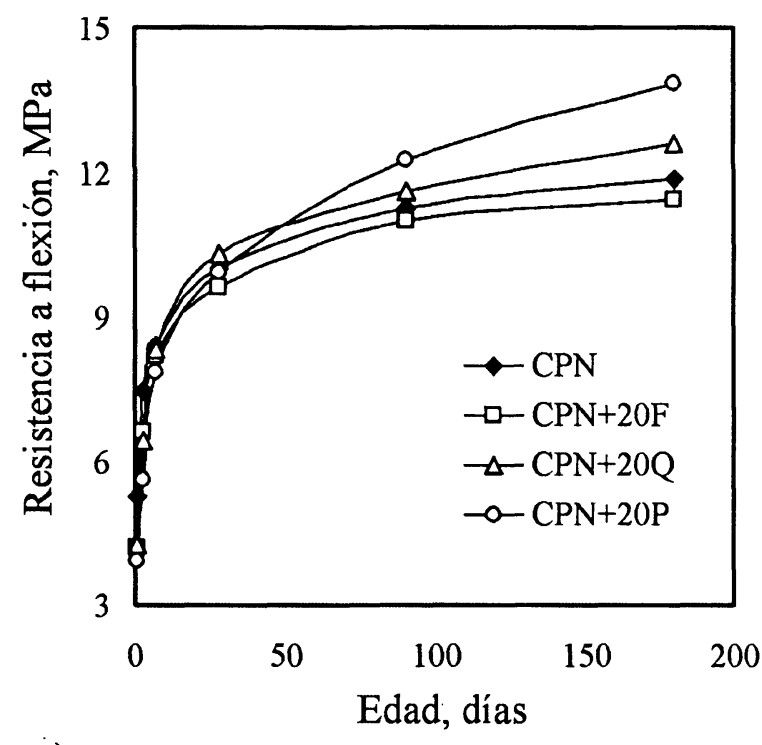

a)

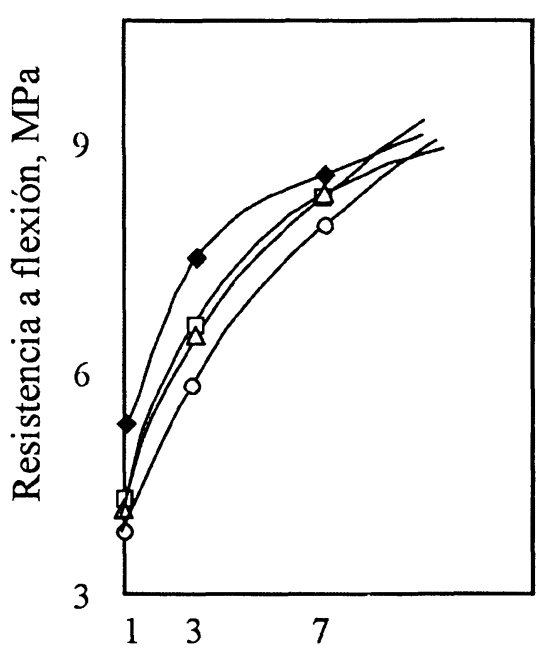

Edad, días

b)

Figura 3.- a) Resistencia a flexión de las pastas y b) Ampliación entre 1 y 7 días.

$\mathrm{CPN}+20 \mathrm{Q}$ presentó un comportamiento similar al de la pasta CPN+20F hasta los 7 días, a los 28 días alcanzó la resistencia del patrón y, a partir de esta edad, comenzó a superarla levemente (6\% a los 180 días). La pasta $\mathrm{CPN}+20 \mathrm{P}$ registró las mayores pérdidas de resistencia hasta la edad de 7 días (hasta 26\%); sin embargo, a los 28 días alcanzó la resistencia de la pasta de control y a 180 días la superó en $16 \%$.

En la Figura 4 se puede observar que hasta los 7 días, la resistencia a compresión de la pasta $\mathrm{CPN}+20 \mathrm{~F}$ es hasta $10 \%$ mayor que la correspondiente a la pasta de control, a partir de los 28 días, edad en la que se iguala con el patrón, comienza una disminución gradual de la resistencia alcanzando a ser a los 180 días de $5 \%$ menor. El comportamiento registrado por la pasta $\mathrm{CPN}+20 \mathrm{Q}$, al igual que lo manifestado en la resistencia a flexión, fue similar al de la pasta $\mathrm{CPN}+20 \mathrm{~F}$ en las primeras edades. Sin em- bargo, a partir de los 28 días se produce un leve incremento de la resistencia con respecto al control, llegando a ser del $5 \%$ a los 180 días. Por último, la pasta $C P N+20 P$ alcanzó a los 180 días un aumento del $20 \%$ con respecto a la pasta de control.

Tanto la resistencia a flexión como a compresión registradas por las muestras, puede ser explicada a través de los resultados del agua no evaporable, pues este parámetro es un buen indicador del progreso de la reacción de los silicatos de calcio del cemento.

La Figura 5 muestra la relación entre la resistencia a compresión y el contenido de agua no evaporable de las pastas de cemento mezcla. En la misma puede observarse que para alcanzar un mismo nivel resistente fue necesario incrementar el contenido de agua no evaporable de las pastas $\mathrm{CPN}+20 \mathrm{~F}$ y $\mathrm{CPN}+20 \mathrm{Q}$, para poder compensar el efecto de dilución del clínker portland. 



a)

Figura 4.- a) Resistencia a compresión de las pastas y b) Ampliación entre 1 y 7 dias.



Agua no evaporable

Figura 5.- Contenido de agua no evaporable versus la resistencia a compresión de las pastas.

Por el contrario y a edades avanzadas de la hidratación, el mismo nivel resistente entre el control y la muestra $\mathrm{CPN}+20 \mathrm{P}$ puede alcanzarse con un menor contenido de agua no evaporable de esta última. Este comportamiento se debe fundamentalmente al refinamiento de poros y granos, que la puzolana produce durante su reacción.

En las pastas con $\mathrm{CPN}+20 \mathrm{~F}$, si bien siempre existe mayor cantidad de agua no evaporable que en la pasta de control, esto no se traduce en un aumento directo sobre las propiedades resistentes, debido a que el volumen de productos de hidratación con características cementantes en el cemento con caliza molida es menor que en el cemento portland normal. A pesar de que esta adición no es inerte, pues está ampliamente comprobada la reacción del $\mathrm{C}_{3} \mathrm{~A}$ con el $\mathrm{CaCO}_{3}$ para dar monocarboaluminato de calcio hidratado (3), no produce C-S-H, que es el compuesto que en mayor medida aporta resistencia a la pasta. En la pasta $\mathrm{CPN}+20 \mathrm{Q}$, el aumento inicial de la resistencia puede justificarse por el aumento en la cantidad de agua no evaporable ocasionado por el efecto filler, en tanto que el aumento posterior de estos parámetros se deba a una reacción puzolánica que se desarrolla en la superficie del cuarzo (7-9), la cual ha sido verificada por el ensayo de puzolanicidad (Figura 2).

Por último, en la pasta $\mathrm{CPN}+20 \mathrm{P}$, a edades tempranas también se manifiesta el efecto filler, pero el efecto de dilución es predominante, en consecuencia la resistencia mecánica y el agua no evaporable resultan menores que en la pasta de control. Un comportamiento similar, también ha ocurrido en morteros con cemento y $20 \%$ de escoria de alto horno. Con el transcurso de la hidratación y de la reacción de la escoria, aumentan la resistencia, debido al 
refinamiento de poros y de granos, y la cantidad de agua no evaporable por el incremento en el contenido de C-S-H (14).

Difracción por rayos X: la Figura 6 muestra la evolución de los productos de hidratación de las pastas identificados por DRX, a las edades de 1, 3, 7, 28 y 90 días.

En la Figura 6a se observan los difractogramas de la pasta con cemento CPN. En la misma puede observarse que a un día los principales productos de hidratación detectados son ettringita y $\mathrm{CH}$. A partir de los 3 días, la ettringita no permanece estable y parte de ella se transforma en monosulfoaluminato de calcio hidratado, registrándose un incremento en la cantidad de este compuesto hasta la edad de 90 días. Por otro lado, la conversión de ettringita a monosulfoaluminato de calcio hidratado se debió a que el cemento portland empleado en este trabajo (relación molar $\mathrm{SO}_{3} / \mathrm{C}_{3} \mathrm{~A}=1.5<3$ ) presentó una deficiencia de iones sulfatos con relación al contenido de $\mathrm{C}_{3} \mathrm{~A}(15)$.
Por otra parte, en la Tabla 1 se muestran los resultados obtenidos del análisis semicuantitativo del pico de $\mathrm{CH}$ $(\mathrm{d}=4,90 \mathrm{~nm})$ para las distintas pastas. En la misma se puede ver que en la pasta con CPN se produce un incremento gradual de la cantidad de este compuesto a todas las edades, evidenciando la progresiva reacción de los silicatos de calcio del clínker portland.

Los difractogramas de las pastas $\mathrm{CPN}+20 \mathrm{~F}$ se muestran en la Figura 6b. A un día se detectó la formación de ettringita y $\mathrm{CH}$, en tanto que a tres días también pudo apreciarse el monocarboaluminato de calcio hidratado. La ettringita permaneció estable hasta los 7 días y después comenzó a convertirse en monosulfoaluminato de calcio hidratado, el cual sólo fue detectado a la edad de 28 días, apareciendo nuevamente la ettringita a los 90 días. Entre los 7 y 28 días los picos correspondientes al monocarboaluminato de calcio hidratado permanecieron aproximadamente constantes. A partir de esta última edad

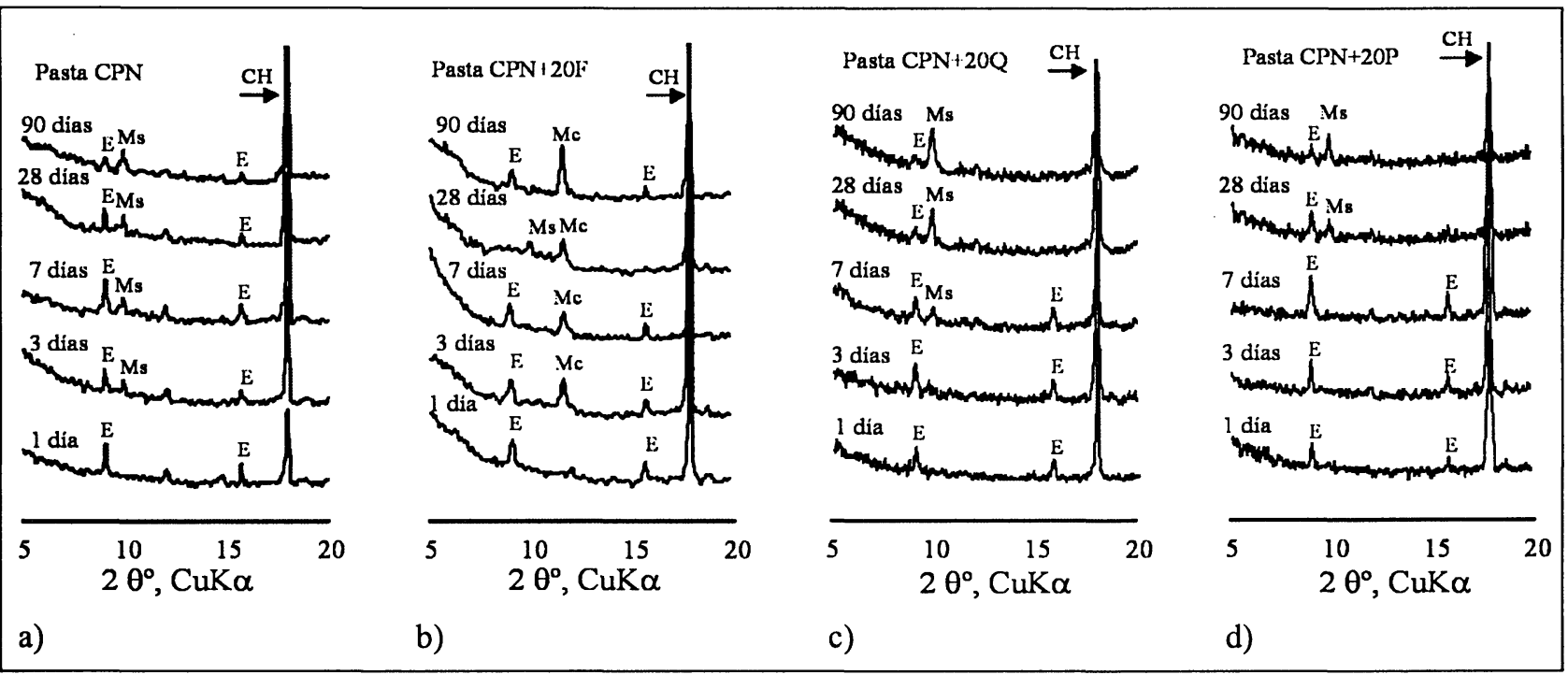

Figura 6.- Difractogramas de las pastas de cemento mezcla. a) $\mathrm{CPN}$, b) $\mathrm{CPN}+20 \mathrm{~F}$, c) $\mathrm{CPN}+20 \mathrm{Q}$ y d) $\mathrm{CPN}+20 \mathrm{P}$. E: ettringita, Ms: monosulfoaluminato de calcio hidratado, $\mathrm{CH}$ : hidróxido de calcio, Mc: monocarboaluminato de calcio hidratado.

TABLA 1

Análisis semicuantitativo del pico de hidróxido de calcio

$\left(2 \theta=18,09^{\circ}, \mathrm{d}=4,90 \mathrm{~mm}\right)$

Cantidad de $\mathbf{C H}$, cuentas/s

\begin{tabular}{|c|cccc|}
\hline Edad & CPN & CPN+20F & CPN+20Q & CPN+20P \\
\hline 1 & 146.2 & 174.5 & 166.1 & 125.9 \\
3 & 225.4 & 276.6 & 272.3 & 195.3 \\
7 & 241.5 & 296.9 & 284.1 & 223.8 \\
28 & 336.4 & 355.0 & 328.0 & 259.2 \\
90 & 367.9 & 381.1 & 292.1 & 208.3 \\
\hline
\end{tabular}


la cantidad de monocarboaluminato de calcio hidratado se incrementó considerablemente. Este comportamiento fue analizado en un trabajo previo (1), en el cual se mostró que la conversión inicial de ettringita a monosulfoaluminato de calcio hidratado, ocurrió debido a que la cantidad de yeso en la pasta fue deficiente y, posteriormente, la conversión de monosulfoaluminato de calcio hidratado a ettringita ocurrió por el aportc de iones sulfatos liberados por ${ }^{*}$ la transformación del monosulfoaluminato de calcio hidratado a monocarboaluminato de calcio hidratado.

Por último, la evolución del pico de $\mathrm{CH}$ de la pasta CPN+20F pucde observarse en la Tabla 1. De clla se desprende que, a las primeras edades, la cantidad de $\mathrm{CH}$ de la muestra $\mathrm{CPN}+20 \mathrm{~F}$ es considerablemente mayor que en la muestra CPN, alcanzando un incremento a los 7 días de un $23 \%$, a pesar de que la muestra tiene un $20 \%$ menos de cemento portland. A partir de los 28 días este comportamiento se revierte y el aumento de la cantidad de $\mathrm{CH}$ apenas llega al $4 \%$ a los 90 días. Los resultados obtenidos, permiten corroborar, una vez más, la presencia de dos efectos antagónicos como son el efecto filler y el efecto de dilución.

Los espectros de DRX de las pastas CPN+20Q se muestran en la Figura 6c. Los productos de hidratación identificados hasta los 3 días fueron: ettringita y $\mathrm{CH}$. A partir de csta edad se puede observar la transformación de ettringita en monosulfoaluminato de calcio hidratado, la cual continúa hasta la edad de 90 días, debido a la deficiencia de iones sulfatos en el sistema. La cantidad de $\mathrm{CH}$ (Tabla 1) liberado por la pasta $\mathrm{CPN}+20 \mathrm{Q}$ hasta los 7 días fue similar a lo registrado por la pasta $\mathrm{CPN}+20 \mathrm{~F}$. Sin embargo, a partir de los 28 días, este parámetro disminuyó considerablemente con respecto a las pastas $\mathrm{CPN}+20 \mathrm{~F}$ y CPN, evidenciando el consumo de $\mathrm{CH}$ por la reacción puzolánica.
En la Figura 6d se muestran los difractogramas correspondientes a la pasta CPN+20P. En la misma puede observarse que, hasta los 7 días, los productos de hidratación detectados son: ettringita y $\mathrm{CH}$. A partir de esta edad la ettringita comenzó a transformarse a monosulfoaluminato de calcio hidratado. Aun así, la ettringita presenta una mayor estabilidad que la registrada en la pasta con cuarzo, pues su transformación se verifica posteriormente. Complementariamente, en la Tabla 1 se puede observar la evolución del $\mathrm{CH}$ obtenido por el análisis semicuantitativo. La cantidad de $\mathrm{CH}$ registrada por la muestra $\mathrm{CPN}+20 \mathrm{P}$ a todas las edades fue menor que la correspondiente a la pasta CPN. Resultando las cantidades medidas hasta los 7 días superiores a las que se hubicran obtenido con $80 \%$ de cemento portland, evidenciando la presencia del efecto filler. Después de los 28 días, la cantidad de $\mathrm{CH}$ disminuyó considerablemente mostrando el consumo de este compuesto por la reacción de la puzolana.

\section{CONCLUSIONES}

De la experiencia realizada con los materiales seleccionados y en las proporciones utilizadas, se pueden obtener las siguientes conclusiones:

- La incorporación de adiciones minerales finamente divididas produce una aceleración de las reacciones de hidratación del clínker portland a tempranas edades.

- Si la adición durante la hidratación de la pasta de cemento no genera C-S-H, el efecto de dilución se hace presente a edades tardías, provocando una disminución de la resistencia final.

. La incorporación de cuarzo finamente molido al clínker portland produce a largas edades una reacción con el $\mathrm{CH}$ produciendo nuevos productos de hidratación.

\section{BIBLIOGRAFÍA}

(1) V.L. Bonavetti, V.F. Rahhal, E.F. Irassar. Studies on the carboaluminate formation in limestone filler blend. Cement and Concrete Research, Vol. 31, No 6 (2001), pp. 883-859.

(2) R. Feldamn, V. Ramachandran, P. Sereda. Influence of $\mathrm{CaCO}_{3}$ on the Hydration of $\mathrm{C}_{3} A$. Journal of the American Ceramic Society. Vol. 48, No 1 (1965), pp. 25-30.

(3) V.L. Bonavetti. Limestone filler cements: Interaction mechanism and its influence on mechanical properties. MSc thesis, Universidad Nacional del Centro de la Provincia de Buenos Aires, Argentine, (1998), 242 p.

(4) V. Rahhal, R. Talero. Análisis de la influencia de las adiciones cristalinas sobre la hidratación de los cementos portland. Anales de la Asociación Química Argentina. En prensa (2002).

(5) V.L. Bonavetti, V.F. Rahhal. Morteros de cemento portland con adición de filler calizo. Hormigón. № 30 (1996), pp. 37-48.

(6) A.M. Paillère, M. Raverdy. L' influence d'ajouts Inertes ou Actifs sur les Propiétés des Ciments. Bulletin des Laboratoires des Ponts et Chaussées. No 90 (1977), pp. 49-60.

(7) F. De Larrard. Formulation et propriétés des bétons à très hautes performances. Laboratoire Central des Ponts et Chausscées. Rapport de recherche LCP Nº 149 (1988), p. 37.

(8) F. Massazza, M. Pezzuoli. Cement paste-quartz bond in autoclaved concretes. Proc. 7th International Congress of the Chemistry of Ccment. París, Francia. Vol. VII (1980), pp. 16-21. 
(9) G.P. Tognon, S. Cangiano. Interface phenomena and durability of concrete. Proc. 7th International Congress of the Chemistry of Cement. París, Francia. Vol. VII (1980), pp. 133-138.

(10) C. Zhang, A. Wang, M. Tang, X. Liu. The filling role of pozzolanic material. Cement and Concrete Research, Vol. 26, $\mathrm{N}^{\circ} 6$ (1996), pp. 943-947.

(11) A.M. Neville, J.J. Brooks. Tecnología del Concreto. Editorial Trillas SA. Mćxico. (1998).

(12) T.C. Powers. The non evaporable water content of hardened portland cement paste. ASTM Bulletin. No 158 (1949), pp. 68-75.

(13) l. Opoczky. Progress of the particle size distribution during the intergrinding of a clinker-limestone mixture. Zement-Kalk-Gips. Vol. 45, No 12 (1992), pp. 648-651.

(14) G. Menéndez, V. Bonavetti. E.F. Irassar. Ternary blended cement containing limestone filler and slag. Proc. International Conference on Sustainable Construction into the Next Millennium: Environmentally Friendly and Innovative Cement Based Materials. Edited by N. Barbosa, R. Swamy and C. Lynsdalc. João Pessoa, Brazil, Noviembre (2000), pp. 188-199.

(15) F. Zhang, Z. Zhou. Z. Lou. Solubility product and stability of ettringite. Proc. 7th International Congress of the Chemistry of Cement. París, Francia. Vol. II (1980), pp. 88-93.

\section{Publicaciones del Instituto Eduardo Torroja - CSIC}

\section{Números monográficos de INFORMES}

\begin{tabular}{|l|l|}
\hline & $\begin{array}{l}\text { Santiago Capital } \\
\left(n^{\circ} 438\right)\end{array}$ \\
& - El color a las puertas de Santiago. \\
& - Un nuevo camino en Santiago, el periférico de Compostela. \\
& - Paso superior del enlace de Fontiñas en la nueva red periférica de Santiago de Compostela. \\
& Retorno a Stonehenge. \\
& - El puente de La Rocha en Santiago de Compostela: Ampliación de dos a cuatro carriles de \\
& circulación del puente existente en el KM 67 de la CN-550 de La Coruna a Santiago y Tuy. \\
& - Nuevo mercado nacional de Ganado. \\
& - Obras de rehabilitación en el mercado de abastos de la ciudad histórica de Santiago de \\
& Compostela. \\
& - Dos proyectos de urbanización en Santiago. \\
& - Palacio multiusos del Sar.
\end{tabular}

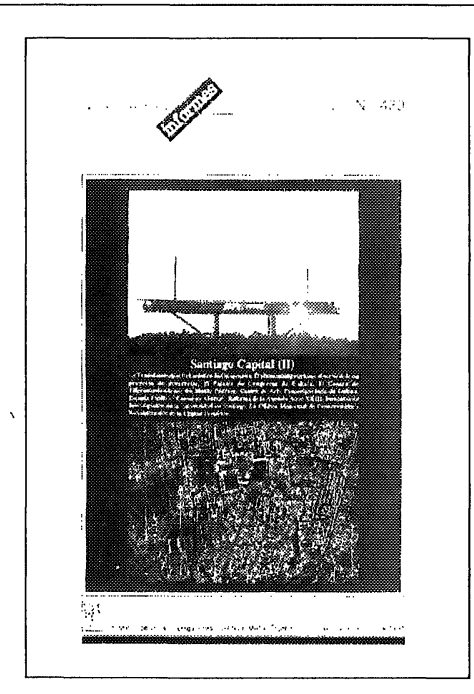

$\left(n^{\circ} 439\right)$

- La transformación urbanística de Compostela. El planeamiento urbano al servicio de un proyecto de desarrollo (Galicia-España).

- Palacio de Congresos y Exposiciones de Galicia en Santiago (Galicia-España).

- Nueva instalación de Telecomunicaciones. Santiago de Compostela (Galicia-España).

- Centro de Arte Contemporáneo de Galicia en Santiago de Compostela (Galicia-España).

- Escuela Pública "Carme de Abaixo". Santiago de Compostela (Galicia-España).

- Avenida Xoan XXIII. Santiago de Compostela (Galicia-España).

- Edificios para Instituto de Investigación de la Universidad de Santiago. Santiago de Compostela (Galicia-España).

- La Oficina Municipal de Conservación y Rehabilitación de la Ciudad Histórica de Santiago de Compostela (Galicia-España). 